\title{
A rare and forgotten cause of aortic aneurysm: tertiary syphilis
}

\author{
Carmen Quiñonez ${ }^{1}$, Nathalia Buitrago-Gómez ${ }^{2}$, Jose Oñate ${ }^{1}$, Raul Castillo ${ }^{1}$, Carlos Devia ${ }^{1}$, \\ and Victor Buchelli ${ }^{1}$ \\ ${ }^{1}$ Centro Médico Imbanaco \\ ${ }^{2}$ Universidad Libre - Campus Cali
}

April 20, 2021

\begin{abstract}
Cardiovascular syphilis occurs 15 to 30 years after infection. Syphilitic aortitis is the most common manifestation and typically involves the ascending aorta. Aneurysm formation is the least common complication of aortitis; the majority of aneurysms are single and usually saccular. We report the images of a 51-year-old man with a history of chest pain that began one month before. An aneurysm of the ascending aorta was documented. The nontreponemal and treponemal tests were reactive. Other venereal diseases, as well as neurological involvement, were discarded. The patient received treatment for non-neurologic tertiary syphilis and surgical repair by the Bentall procedure with re-implantation of the coronary arteries. Pathology showed the aortic arterial layer displaying myxoid changes in the tunica intima, as well as inflammatory infiltrate. The vasa vasorum demonstrates inflammatory infiltration with lymphocytes and plasma cells. The previous findings support the diagnosis of syphilitic aortitis. The patient's evolution was satisfactory with no current cardiovascular symptoms.
\end{abstract}

\section{Hosted file}

study syphilis 18:04.pdf available at https://authorea.com/users/409011/articles/518800-arare-and-forgotten-cause-of-aortic-aneurysm-tertiary-syphilis 
figures/Img-09/Img-09-eps-converted-to.pdf 
figures/Img-04/Img-04-eps-converted-to.pdf 
figures/Img-07/Img-07-eps-converted-to.pdf 